\title{
Educación comunitaria: una propuesta alternativa para los pueblos indígenas de Oaxaca - México
}

\author{
Arturo Ruiz López \\ Universidad Autónoma Benito Juárez de Oaxaca, Oaxaca, México. \\ Email: rarturol@hotmail.com \\ Elena Quiroz Lima \\ Universidad Pedagógica Nacional, Oaxaca, México. \\ Email: quirozelena@gmail.com
}

\begin{abstract}
Resumen: La educación para los indígenas en México ha pasado por diferentes etapas y ha tenido distintos objetivos. Con la globalización económica y cultural, las escuelas situadas en comunidades indígenas enfrentan ahora nuevos retos, como es atender y respetar la diversidad cultural conservando la cultura originaria. En este contexto, las Secundarias Comunitarias (SC) han surgido en el Estado de Oaxaca - México como una propuesta educativa con un enfoque intercultural, centrándose en la comunidad para el desarrollo de los contenidos escolares. La implementación de esta propuesta devela el desarrollo de una actitud crítica por parte de los estudiantes que fortalecen su identidad étnica. Sin embargo, también presenta limitaciones en la construcción de conocimientos en el ámbito de las disciplinas exactas, por lo cual requiere de una revisión más amplia.
\end{abstract}

Palabras clave: educación, equidad, comunidad, indígenas

\section{Community education: an alternative proposal for indigenous people in Oaxaca - Mexico}

\begin{abstract}
Education for indigenous people in Mexico has had several stages and different objectives. With the economic and cultural globalization, the schools in indigenous communities now face new challenges, such as meeting and respecting cultural diversity preserving the original culture. In this context, the Community Middle Schools (SC) have emerged in Oaxaca-México with an educational proposal with an intercultural approach focussing on the community for the development of classroom content. The implementation of this proposal revels the development of a critical attitude on the part of students who strengthen their ethnic identity. However, it also has limitations in the construction of knowledge concerning the exact disciplines, which requires further review.
\end{abstract}

Key words: education, equity, community, indigenous 


\section{Educação Comunitária: uma proposta alternativa para os povos indígenas de Oaxaca - México}

Resumo: A educação para os povos indígenas no México passou por várias etapas e teve objetivos diferentes. Com a globalização econômica e cultural, as escolas situadas em comunidades indígenas enfrentam agora novos desafios, tais como atender e respeitar a diversidade cultural preservando a cultura originaria. Neste contexto, as Escolas Secundarias Comunitárias (SC) surgiram no Estado de Oaxaca - México com um enfoque educacional com uma abordagem intercultural, centrado na comunidade para o desenvolvimento de conteúdos em sala de aula. A implementação desta proposta revela o desenvolvimento de uma atitude crítica dos estudantes que visam fortalecer sua identidade étnica. No entanto, também tem limitações na construção de conhecimentos no campo das disciplinas exatas, o que requer uma revisão posterior mais abrangente.

Palavras-chave: educação, equidade, comunidade, indígenas.

$* * *$

\section{Introducción}

El presente trabajo recupera la experiencia y los testimonios de los actores principales de un modelo educativo conocido en México como Secundaria Comunitaria (SC). El objetivo es mostrar la aplicación y desarrollo de esta propuesta educativa y su impacto en los estudiantes, padres de familia y autoridades comunitarias en San Andrés Solaga, una localidad de la Sierra Norte de Oaxaca donde realizamos investigaciones entre 2009 y 2012. Varias visitas a la comunidad permitieron recopilar información a través de observaciones, registro de aula y entrevistas cualitativas a los diferentes actores involucrados ${ }^{1}$. El texto se organiza en cuatro apartados cuya articulación se sustenta en las interrogantes que guiaron esta investigación: ¿cómo surge el modelo de Secundarias Comunitarias?, ¿cuál es su fundamentación teóricometodológica?, ¿cuál es su impacto en la comunidad y su nivel de aceptación? y, finalmente, ¿es un modelo pertinente para las comunidades originarias?

En el primer apartado se propone así una revisión de lo que ha sido la educación rural comunitaria a partir de los años 1920, pasando por los modelos de integración e incorporación de la población indígena a la sociedad nacional, hasta llegar al actual modelo de Educación Bilingüe Intercultural. Destacamos que otros proyectos educativos cobran importancia en este proceso, como son el Sistema Nacional de Promotores y Maestros Bilingües, el Programa de Castellanización para los Niños Indígenas y posteriormente la propuesta de Educación Bilingüe Bicultural.

En el siguiente apartado, se aborda el modelo de "Método de Proyectos” que sustenta la propuesta de Secundaria Comunitaria. Se señalan los elementos que lo conforman, como la indagación, la lectura, 
la escritura y la oralidad, tanto en la lengua originaria como en español, competencias que desarrollan los alumnos a largo de su proceso de formación en las Secundarias Comunitarias, además de la revalorización de los conocimientos comunitarios, reforzando en ellos el sentido de comunalidad y pertenencia. Actualmente existen diez Secundarias Comunitarias, localizadas en diferentes regiones del Estado.

El tercer apartado se enfoca más específicamente a la experiencia de San Andrés Solaga. Recogiendo las opiniones de los coordinadores de grado, alumnos, padres de familia y Autoridades Comunitarias sobre la pertinencia y viabilidad del modelo educativo, se analiza la forma en que los propios actores han interiorizado el modelo de SC.

El último apartado cierra el artículo con comentarios generales, en donde manifestamos que la SC se construye “desde abajo", proponiendo una ruptura con esquemas homogenizantes y verticales, en consideración de las necesidades y de los contextos de las comunidades originarias de México. Así también, señalamos algunos vacios detectados por los propios alumnos. Al ser una propuesta alternativa en México, insistimos finalmente en la importancia de darle seguimiento, reflexionar sobre sus alcances y valorar los avances que permitiría en materia de educación para los pueblos originarios.

\section{Antecedentes de la educación comunitaria}

La educación comunitaria encuentra sus antecedentes en los planteamientos de Paulo Freire (1999), quién considera que la educación debe llevarse a cabo en los contextos vivenciales, para la formación de un ciudadano autónomo. Por lo tanto, se propone establecer una relación comprometida del profesor con la comunidad y estrechar lazos directos con la realidad que contextualiza a la escuela (Pérez y Sánchez 2005). La estrategia metodológica implica que profesores y alumnos problematicen una situación concreta y objetiva para que, captándola críticamente, actúen sobre ella. En este proceso, el pensamiento, lenguaje y el contexto se interrelacionan de forma permanente, porque el pensamiento es ante todo un acto colectivo (Freire 2007).

En México, la educación comunitaria tiene sus orígenes en la escuela rural mexicana, con el maestro Rafael Ramírez. Esta escuela debía proporcionar una educación integral, identificada con las necesidades de las comunidades rurales, enseñando la lectura, la escritura y las operaciones fundamentales, pero también aspectos relacionados con la vida de estas comunidades y con las necesidades de sus familias (Rangel 2006). Muchas de estas escuelas se establecieron en comunidades indígenas.

La educación para los pueblos indígenas, específicamente, ha transitado por diferentes etapas. En la década de 1920, se promovió en 
las escuelas el uso exclusivo del español durante el proceso de enseñanza-aprendizaje. Esta propuesta educativa se conoce como Método Directo. Sin embargo, dicho método no dio los resultados esperados y, en los años 1930, se incorporó una nueva propuesta: el Método Indirecto, que plantea el uso de la lengua originaria como punto de partida para llegar al español como lengua meta (Ruiz 1993). Pese a su diferencia formal, ambas propuestas se encaminaban hacia la construcción de un Estado-nación homogéneo. En 1963, el Método Indirecto se inicia con el reclutamiento de jóvenes indígenas hablantes nativos como Promotores Culturales o Maestros Bilingües. Con este proyecto se buscó integrar el uso de lenguas indígenas en el proceso de aprendizaje, junto con la realización de actividades extraescolares fuertemente vinculadas a la comunidad.

A finales de los 1970, el Método Indirecto fue desplazado por la implementación de un modelo de castellanización para las comunidades indígenas, que fue fuertemente criticado y cuestionado desde la antropología, hasta calificarlo como una forma de etnocidio (Bonfil Batalla 1982). Fue en este contexto que, a mediados de los 1980, surgió el modelo bilingüe bicultural, que pretendió, a partir de lo que hemos llamado documentos básicos (Ruiz 1993), establecer una educación bilingüe usando de manera “equilibrada” dos lenguas, la originaria y el español. En cuanto a lo bicultural, se refiere a la recuperación de contenidos comunitarios y su integración al modelo oficial de educación, dando finalmente lugar en 2001 al modelo de Educación Indígena Bilingüe Intercultural (Ruiz 2002).

La incorporación de la educación intercultural en las propuestas educativas ha evolucionado poco a poco hasta cobrar gran relevancia, especialmente en los contextos indígenas. En Latinoamérica, existen varias experiencias, como en Perú, por ejemplo, donde se implementó un proyecto conjunto entre el Ministerio de Educación y la Asociación Interétnica para el Desarrollo de la Selva Peruana (AIDESEP), para la formación de profesores que contempla una perspectiva intercultural. Esta perspectiva sitúa a los niños y niñas, a sus comunidades y manifestaciones lingüísticas y culturales, en el eje principal de la formación docente para el desarrollo sostenible de las comunidades (Quiroz 2005). Durante el II Congreso Nacional de la Educación Indígena e Intercultural, realizado en 2007 en Oaxaca - México, se propuso cuatro líneas de acción (ver Tabla $N^{\circ} 1$ ), las cuáles se tomarían en cuenta posteriormente para reconsiderar algunos aspectos de la propuesta educativa de las Secundarias Comunitarias. 


\section{Tabla N¹: Líneas de acción del II Congreso de Educación Indígena e Intercultural, 2007}

\begin{tabular}{|l|l|l|}
\hline \multicolumn{2}{|c|}{ LINEAS } & \multicolumn{1}{c|}{ ACCIONES } \\
\hline 1 & $\begin{array}{l}\text { Creación de propuestas } \\
\text { educativas }\end{array}$ & $\begin{array}{l}\text { Los pueblos indígenas, mediante sus diferentes actores, } \\
\text { definirán las propuestas educativas para sus hijas e hijos, } \\
\text { recibiendo de los maestros y autoridades educativas apoyo } \\
\text { profesional. }\end{array}$ \\
\hline 2 & $\begin{array}{l}\text { Revalorar las lenguas } \\
\text { originarias }\end{array}$ & $\begin{array}{l}\text { Identificary revalorar críticamente en las escuelas las lenguas, } \\
\text { los saberes y las prácticas de los pueblos originarios. }\end{array}$ \\
\hline 3 & $\begin{array}{l}\text { Formación de } \\
\text { profesores }\end{array}$ & $\begin{array}{l}\text { Reorientar la formación y capacitación de maestros para } \\
\text { priorizar los valores y las prácticas comunales, e iniciar una } \\
\text { red de formadores para capacitar a maestros indigenas } \\
\text { seleccionados, quiénes serán los futuros formadores. }\end{array}$ \\
\hline 4 & $\begin{array}{l}\text { Formación comunal de } \\
\text { ciudadanos }\end{array}$ & $\begin{array}{l}\text { Crear un centro nacional de formación comunal multilingüe e } \\
\text { intercultural como un espacio en el que se promueva la } \\
\text { comunalidad, el multilingüismo y la interculturalidad, con el } \\
\text { propósito de capacitar a educadores comumales (sean o no } \\
\text { maestros en servicio) con las competencias necesarias para } \\
\text { investigar y analizar las realidades de sus comunidades y } \\
\text { generar prácticas didácticas apropiadas. }\end{array}$ \\
\hline
\end{tabular}

Fuente: elaboración propia con información de Meyer (2010).

Las líneas de acción reconocen la participación de los diferentes actores de los pueblos indígenas en la definición de las propuestas educativas para sus hijas e hijos. Asimismo, se reconoce la necesidad de recibir apoyo profesional de profesores especialistas y de las autoridades educativas. Lo anterior cobra mucho sentido en el caso de Oaxaca, tratándose de uno de los Estados mexicanos con mayor diversidad cultural. En su territorio conviven 16 grupos etnolingüísticos y el 33.8\% de su población habla alguna lengua indígena (INEGI 2010). La diversidad cultural y lingüística representa un gran reto para el sector educativo del Estado, por lo que la búsqueda de un modelo que responda a las necesidades e intereses de las comunidades indígenas ha sido una constante. Sin embargo, la interculturalidad es un campo aún emergente en la investigación académica, en planeación política e institucional, así como en la intervención pedagógica (Dietz y Mateos 2011).

En 2001, durante el gobierno del presidente Vicente Fox, se creó en México la Coordinación General de Educación Intercultural y Bilingüe (CGEIB), la cual tiene como misión "contribuir a la construcción de una sociedad más justa y equitativa, mejorando y ampliando las oportunidades educativas de la población indígena y promoviendo relaciones más igualitarias y respetuosas entre los integrantes de toda la sociedad mexicana"2. Con estos antecedentes, surgió en Oaxaca el año 2004 el proyecto alternativo denominado Secundaria para la Atención de Comunidades Pertenecientes a los Pueblos Originarios del Estado de Oaxaca, el cual se cono- 
ce como Secundarias Comunitarias (SC). El proyecto fue impulsado por diferentes instancias: la Coalición de Maestros y Promotores Indígenas de Oaxaca del Instituto Estatal de Educación Pública de Oaxaca, la Sección XXII del Magisterio oaxaqueño, y la CGEIB.

\section{La propuesta de la Secundaria Comunitaria}

El documento base de las Secundarias Comunitarias incluye los lineamientos, marco conceptual, objetivos y requerimientos necesarios para llevar a cabo la propuesta. La SC se concibe como una estructura de la comunidad, la cual tiene la misión de contribuir a la preservación, fortalecimiento y enriquecimiento de la cultura originaria. Además, se espera que las SC promuevan la mejora de la calidad de vida comunitaria; el desarrollo humano de los miembros de la comunidad; y ayuden a las nuevas generaciones a concluir su educación básica (IEEPO 2004).

La propuesta de SC se implementó inicialmente en cinco escuelas localizadas en diferentes comunidades de Oaxaca: 1) San Andrés Solaga: 2) Santa María Tiltepec; 3) San Pedro Yaneri; 4) Tlalixtac Viejo y 5) Arroyo Blanco. Posteriormente, se sumaron las comunidades de San Juan Teotalcingo y Santa María Zoogochí, alcanzando así un total de diez escuelas en 2013. Las SC se instalan en lugares marginados que requieren educación para los adolescentes que no tienen opción cerca de su comunidad de origen por no reunir el número necesario de alumnos para establecer una secundaria de organización completa, uno de los requisitos más importantes ${ }^{3}$. Las comunidades, por su parte, pueden respaldar la instalación y funcionamiento de las SC mediante un acuerdo que se firma en Asamblea Comunitaria. Pero al no contar con el respaldo total del Instituto Estatal de Educación Pública de Oaxaca (IEEPO) ${ }^{4}$ para la instalación de las SC, los padres de familia suelen resolver problemas de infraestructura, económicos y humanos por otras vías.

La SC reconoce la diversidad cultural del Estado y favorece la expresión de las manifestaciones culturales en las aulas. Quizá la más importante es la valoración y estimulación para que los estudiantes se expresen en lengua originaria dentro de las aulas. Asimismo, se promueve la revalorización de los conocimientos comunitarios, reforzándose en los estudiantes el sentido de comunalidad (IEEPO 2004; Maldonado 2002; Jiménez 2009). La SC tiene varias características (Tabla N² 2) que podemos agrupar en tres grandes categorías: pedagógicas, comunalidad e interculturalidad. 
Tabla N²: Características de la Secundaria Comunitaria

\begin{tabular}{|c|c|}
\hline CARACTERISTICAS & ACCIONES \\
\hline Pedagógicas & $\begin{array}{l}\text {-Toma de decisiones colectiva } \\
\text {-Respeto a la libertad y la autonomía, así como generar } \\
\text { la reflexión } \\
\text {-Participación igualitaria en el ámbito escolar } \\
\text {-Concepción del alumno como centro de la práctica } \\
\text { educativa } \\
\text {-Concepción del educador como facilitador del } \\
\text { conocimiento } \\
\text {-Implementación del método de proyectos } \\
\text {-Evaluación concebida de manera integral y cualitativa }\end{array}$ \\
\hline Comunalidad & $\begin{array}{l}\text {-Ser parte integral de la comunidad } \\
\text {-Parte de conocimientos, valores y tecnologías } \\
\text { comunitarias }\end{array}$ \\
\hline Interculturalidad & $\begin{array}{l}\text {-Uso libre de la lengua originaria } \\
\text {-Respeto a la diversidad lingüística y cultural } \\
\text {-Promoción de la igualdad en la diversidad }\end{array}$ \\
\hline
\end{tabular}

Fuente: elaboración propia con información del IEEPO (2004).

La fundamentación pedagógica de la SC se encuentra en el Método de Proyectos (MP), el cual tiene como principio básico la construcción del conocimiento mediante el establecimiento de hipótesis, la investigación teórica y comprobación empírica (Sánchez 2008)5. Otro principio es el aprendizaje significativo recuperando la propia experiencia de los estudiantes (Ausubel 1976) y fortaleciendo el vínculo permanente de la escuela con la comunidad (Dewey 1998). Con el desarrollo de proyectos se busca promover experiencias de aprendizaje que involucren a los estudiantes en actividades complejas y los lleve a confrontarlas con un contexto real. De esta forma, desarrollan habilidades, aplican conocimientos e intentan solucionar problemas. Una característica particular para el caso de las SC en Oaxaca es la elaboración de textos y la expresión oral en lengua originaria y castellana para recopilar información y analizarla.

De manera general, los proyectos se trabajan en tres fases: apertura, desarrollo y evaluación (ver Tabla $\mathrm{N}^{\circ} 3$ ). Cada una de ellas con actividades específicas para los profesores y alumnos. 


\section{Tabla N³: Desarrollo de los proyectos de investigación en las Secundarias Comunitarias}

\begin{tabular}{|l|l|}
\hline \multicolumn{1}{|c|}{ FASES } & \multicolumn{1}{c|}{ ACTIVIDADES } \\
\hline Apertura & $\begin{array}{l}\text { En la primera fase, el tema o problema a desarrollar se define a } \\
\text { partirdelos intereses de los alumnos, quienes presentan una serie } \\
\text { de preguntas que se priorizan, considerando viabilidad, } \\
\text { pertinenciay relevancia para los alumnos y la comunidad. En esta } \\
\text { etapa se construye el proyecto de aprendizaje o de investigación. }\end{array}$ \\
\hline Desarrollo & $\begin{array}{l}\text { Durante este proceso, sellevan a cabo diferentes actividades para } \\
\text { indagar sobre el tema elegido. Se considera la vinculación con la } \\
\text { comunidad para la investigación, el uso de la biblioteca, Internet } \\
\text { (cuando se cuenta con este servicio), la redacción de informes } \\
\text { parciales y finales. }\end{array}$ \\
\hline Evaluación & $\begin{array}{l}\text { La presentación de resultados en el aula y en la comunidad } \\
\text { mediante la asamblea comunitaria. }\end{array}$ \\
\hline
\end{tabular}

Fuente: elaboración propia.

En el MP, los roles de profesores y alumnos se amplían en comparación con los de la denominada escuela tradicional. Los alumnos se convierten en los sujetos centrales, son ellos quienes llevan a cabo las actividades de forma autónoma en la búsqueda de conocimientos y la construcción de su propio aprendizaje (ITESM s/f). El profesor guía, coordina, orienta y crea ambientes de aprendizaje que permiten a los estudiantes construir su conocimiento, expresar sus dudas y aportaciones. Se puede reconocer que se modifica la relación maestro-alumno, el proceso enseñanza-aprendizaje y la relación escuela-comunidad.

Para el desarrollo de contenidos, se retoman las experiencias y conocimientos de la comunidad y éstos se articulan con los contenidos del Plan y Programas de Educación Secundaria, establecidos a nivel nacional por la Secretaría de Educación Pública. La evaluación corresponde a la tercera fase y se realiza al final del proyecto. La actividad la dirigen los estudiantes, quienes expresan las actividades realizadas, los conocimientos adquiridos y la comprobación o rechazo de los supuestos iniciales con los que empezaron el proyecto. La evaluación se realiza de forma permanente considerando aspectos de tipo cognitivo, de habilidades y actitudes. Los resultados del proyecto se presentan en el aula y en una Asamblea Comunitaria a la que asiste la mayoría de los pobladores de la comunidad. 


\section{La Secundaria Comunitaria en San Andrés Solaga}

La Secundaria Comunitaria de San Andrés Solaga, comunidad ubicada en la región de la Sierra Norte, se fundó en 2004. Inició labores sin contar con aulas, sillas, pizarrones, libros, obligando a los padres de familia a organizarse para obtener recursos económicos y conseguir un espacio físico en el que los alumnos pudieran recibir clases. El primer espacio que les fue asignado fue un anexo al palacio municipal, el cual fue acondicionado con la infraestructura básica. Los profesores encargados de implementar la propuesta de SC fueron seleccionados y capacitados por los coordinadores y diseñadores de la misma. La selección consideró en primer término a los recién egresados de la Escuela Normal Bilingüe Intercultural de Oaxaca, quienes serían los encargados de proporcionar educación secundaria, y trabajar los contenidos de las asignaturas establecidas para éste nivel educativo.

Los profesores señalan que para el primer grado trabajan con un proyecto de aprendizaje, mientras que para los grados de segundo y tercero se trabaja con un proyecto de investigación. La distinción se encuentra en que en el primer grado los estudiantes adquirirán habilidades como la elaboración de entrevistas, búsqueda de información y conocimientos generales para redactar y presentar trabajos. En el segundo y tercer año, con los proyectos de investigación, se acercarán a los saberes y conocimientos comunitarios, por ejemplo las plantas medicinales que se utilizan en la comunidad, las formas de cultivo, los instrumentos musicales de la región, las formas de producción y comercialización, entre otros temas. Estos conocimientos los analizarán y contrastarán con los conocimientos teóricos que se establecen en los programas oficiales y en los diversos documentos que los estudiantes consultan. Los hallazgos de los estudiantes son socializados de manera bilingüe -lengua originaria y español- en los diferentes momentos del desarrollo del proyecto. Los profesores tratan de vincular los contenidos pedagógicos de cada asignatura, pero esto no implica que se impartan por módulos y en horarios específicos, como se hace en las secundarias generales. Los contenidos se revisan según lo requiera el proyecto, tal como lo señala un profesor de segundo grado:

Por ejemplo, el tema de migración en la comunidad nos permite abordar la asignatura de matemáticas con los porcentajes y relacionarlos con los salarios de los migrantes, el envío de remesas, la venta de productos en la comunidad. Los aspectos de historia se revisan con los inicios de la migración, los periodos que se han establecido y se pueden relacionar con otros contextos y eventos como la primera y segunda guerras mundiales. Las asignaturas de redacción se revisan constantemente con la estimulación del lenguaje oral y escrito para comunicar los avances de los proyectos y los resultados finales, los cuales se exponen ante la comunidad (A.M. 2009).

La secundaria comunitaria a través de su práctica pedagógica, cotidianamente promueve el uso y la importancia de la lengua originaria en 
los jóvenes. Algunos de ellos refuerzan la oralidad y aprenden a escribir su lengua originaria, ya que es común que acudan con los ancianos de la comunidad para entrevistarlos sobre diversos temas. De esta forma, los estudiantes reconocen el valor de su lengua originaria, saben que si ellos no la hablan y no la reproducen, se perderá. Lo mismo sucede con la historia, las tradiciones y costumbres. La revalorización comunitaria y local que se lleva a cabo en las SC, se extiende al ámbito familiar con los padres de familia, quienes advierten sobre la importancia de recuperar su lengua vernácula porque se está perdiendo.

Sobre los proyectos que han trabajado ahí, aprenden bastante, porque la vez pasada anduvieron ellos haciendo el proyecto sobre el maíz, el frijol y ahí anduvieron investigando que de dónde provino el maíz, el frijol, hace cuántos años, y ahí veo que [...] viene incluido matemáticas y todo eso, no tiene que ser a fuerzas por materias y todo eso, porque salieron a medir terrenos para ver cuántos almudes de maíz siembran en esos terrenos y al recibir la cosecha qué tanto de maíz recogen y todo eso vieron. Ahorita no estoy muy bien enterada del proyecto, pero sí, veo que es bonito y mi hijo, el otro, aprendió de otra forma, porque ahorita sí, mi hija sí está en la secundaria terminando, de ahí se va al bachillerato porque está aquí también (E.G. 2009).

La forma de trabajar es integral, se promueve el desarrollo de competencias en los alumnos, buscan información cuando lo requieren, buscan en diferentes fuentes de forma oral y escrita y la relacionan con la comunidad. Los estudiantes desarrollan identidad étnica y un sentido de pertenencia a la comunidad reconociendo y valorando sus manifestaciones culturales. Para algunas familias, la SC representa una ventaja económica y la posibilidad de educar a sus hijos de forma más cercana, ya que les permite estar pendientes de su comportamiento:

Mi padre fue presidente municipal cuando se gestionó la secundaria. Ahora sí, que él lo hizo para los alumnos que apenas iban a seguir la secundaria, porque viéndolo bien es un gasto que salen afuera en otros pueblos y estando en la casa hasta uno puede vigilar a los hijos si están asistiendo o no a la escuela, y hay padres que tienen dos, hasta tres, hasta cuatro llegaron a tener en la secundaria cada padre de familia, y fue la ventaja que les ayudó que llegó la secundaria(E.G. 2009).

Esta forma de educación es nueva para los profesores y para la comunidad y aunque la mayoría de la comunidad está de acuerdo con la SC, algunos padres de familia comparan con otras formas de enseñanza. Consideran que la secundaria técnica es mejor porque ahí se enseñan talleres, se dan clases con un profesor por asignatura y se incluye inglés. En la SC no se observa eso, ya que un sólo profesor imparte todas las asignaturas y se habla la lengua originaria. Sin embargo, los profesores de la SC se preguntan: ¿cuánto saben de inglés los alumnos cuando egresan de las secundarias técnicas?, ¿cuánto saben de los talleres que en ellas se imparten?, ¿cuál 
es la pertinencia y relevancia de los conocimientos que se adquieren en las secundarias técnicas?, ¿lo que se aprende sirve para la vida o para resolver problemas concretos e inmediatos en la comunidad? Para responder a estas preguntas, se requiere de mayor análisis, pero sin duda son aspectos que requieren ser revisados.

La SC en Solaga cuenta con seis generaciones y el efecto que ha tenido en la comunidad se observa en la revaloración de la lengua originaria, la historia, las tradiciones y los conocimientos locales. Se han generado varios productos académicos que los estudiantes difunden entre las distintas SC de las diferentes regiones del Estado. Además, han publicado con la coordinación de los profesores, tres textos bilingües (castellano-zapoteco): “El venado, ¿víctima o ser sobrenatural?”; “Solaga, lugar de hojas regadas”; y "La explotación de la mina de San Andrés Solaga”. Las publicaciones resaltan el desarrollo de la escritura en los jóvenes, el análisis, la capacidad investigativa, y muestran que el trabajo de las SC avanza de lo pedagógico hacia una formación de sujetos reflexivos, críticos y sensibles a la vida y problemáticas de su comunidad.

Otros impactos son las acciones gestionadas ante la comunidad a partir de los resultados obtenidos con la implementación de sus proyectos. Uno de ellos se relaciona con la prohibición de la caza del venado cola blanca, hecho que ocurrió después de la presentación de los resultados del proyecto en la Asamblea Comunitaria. En esa exposición, los estudiantes presentaron el abuso de la cacería y las consecuencias a corto y largo plazo. Otro resultado fue la eliminación del uso de bolsas de plástico en las tiendas, comedores y panaderías, invitando a las personas a llevar sus canastas para cargar sus productos y de esta forma reducir la contaminación. Esto se generó después de que los estudiantes presentaran los niveles de contaminación ambiental en la comunidad.

Los egresados de la SC de Solaga reconocen que construyeron competencias que les son de mucha utilidad, entre ellas la de expresarse en público para preguntar o explicar diversos aspectos. Algunos han ingresado a los bachilleratos de la misma región mientras otros se trasladaron a la ciudad de Oaxaca para seguir estudiando. Sobre su experiencia en la SC, los egresados opinan:

Puedes aprender varias cosas, o sea rescatar la cultura, seguir conservando la cultura y seguir investigando lo que se está perdiendo de la comunidad, más que nada... En la Secundaria Comunitaria se trabaja más en la línea de investigación, y para tener los resultados de nuestra línea es pedirles el apoyo a los ancianos de la comunidad y obtener más información de la misma comunidad, y pues seguir renovando lo que hay en las culturas y tradiciones. En mi caso, pues sí me gustó, estudiando allí la secundaria, bueno antes sí fue difícil para mí (por la forma de trabajar), pero ya después conforme pasó el tiempo ya me di cuenta que es bueno estar en una secundaria comunitaria (F.T. 2009). 
El desarrollo de habilidades y competencias permite a los estudiantes enfrentarse a situaciones de su vida cotidiana, por ejemplo investigar por su propia cuenta, trabajar en equipo, ser independientes, autodidactas. Los egresados señalan que algo que aprendieron bien fue investigar y en el bachillerato si no entendían algo lo buscaban ellos mismos en los libros. Sin embargo, también comentan algunas dificultades a las que se enfrentaron al ingresar a los bachilleratos y adaptarse nuevamente al modelo tradicional.

Un modelo comunitario tiene que ver con la comunidad y no viene por materias y sí era necesario que te enseñaran lo que son las materias: química, física, para que cuando entraras al bachillerato no se te hiciera difícil. Que te enseñen inglés porque en la secundaria no llevamos y pues era difícil porque nos empezaron a enseñar en el bachillerato lo que era el abecedario, los números, todo eso ya era más difícil que nos lo aprendiéramos, como en la secundaria no nos enseñaron eso pues, en el bachillerato sí se nos hizo más difícil lo que es el inglés (K.B.S. 2009).

Considerando que los egresados de la SC se van a enfrentar a un contexto nacional e incluso global, algunos contenidos requieren mayor atención para impactar en la formación académica de los estudiantes. En este sentido, los conocimientos básicos en áreas como la física, química, matemáticas y biología, son indispensables para mejorar sus condiciones académicas y poder competir con cualquier otro estudiante para ingresar a las instituciones educativas de nivel bachillerato y superior. De hecho, los estudiantes demandan una profundización sobre estas asignaturas y otras como la enseñanza-aprendizaje del inglés, materia que está ausente del modelo.

Cuando ya eliges una carrera y sabes qué vas hacer, entonces dices: ¿por qué no estudié esto?, ¿por qué lo otro? Entonces, sí, yo diría que metieran un poco más las materias - física, química, biología, matemáticas. Bueno, es lo que yo digo para mejorar un poco la Secundaria Comunitaria, que se centren un poco en lo que son las materias, que no estén investigando solamente sobre la comunidad, sino que también enseñen un poquito de inglés, matemáticas porque eso es lo más importante. Porque cuando yo llegué aquí no vi nada de números, y me costó mucho trabajo, trabajar algebra, trigonometría. Ahorita me está costando mucho trabajo, pues yo no vi eso en la secundaria (F.S. 2009).

Para responder a estas necesidades expresadas por los egresados, los profesores de la SC de Solaga implementaron las Jornadas Académicas al final de cada ciclo escolar, sirviéndoles para profundizar en algunos conocimientos de las asignaturas de las ciencias exactas y ciencias naturales. 


\section{Hacia la mejora de la propuesta educativa}

La SC ha demostrado ventajas en la formación de los estudiantes, ya que ellos construyen diferentes competencias. Además, se les forma cívica y moralmente para poder convivir en la diversidad cultural y lingüística, así como en el respeto hacia la naturaleza. Cuando ellos egresan, estos elementos los ayudan en su vida cotidiana y en su recorrido escolar. También es importante resaltar que este proyecto educativo ha logrado una mayor vinculación e interacción entre los estudiantes y la comunidad; el diálogo permanente que tienen los estudiantes con los ancianos, con los padres de familia, con los informantes clave y con la autoridad local, son muestra de este logro. Todo lo anterior ha permitido reconocer y valorar los conocimientos comunitarios.

Sin embargo, también se enfrentan a grandes retos, ya que cambiar la forma tradicional de trabajar en las escuelas no es sencillo de asimilar para los profesores, alumnos y padres de familia. Asimismo, la SC se concibe como una propuesta educativa integral dentro del sistema de educación formal, lo que implica que se relacione con estructuras y formas de trabajo diferentes que requieren considerar las necesidades de los estudiantes para fortalecer los conocimientos en las áreas de las ciencias exactas y naturales.

La SC requiere que sus profesores tengan una formación multidisciplinaria que no tienen, ya que son en su mayoría egresados de las Escuelas Normales para Educación Primaria que funcionan en el Estado. Por esta razón, es difícil que ellos puedan cubrir todas las necesidades de formación de los estudiantes. Aunque algunos profesores han reconocido sus limitaciones y han buscado asesoría de especialistas que los apoyen impartiendo algunos temas, esto no es suficiente. No hay que olvidar que las comunidades en las que se instalan las secundarias comunitarias son aquellas que presentan condiciones evidentes de marginalidad y que requieren de mayor apoyo. Un solo profesor no da abasto para cubrir todas las asignaturas de un grado. Las SC deben complementarse con profesores especialistas en disciplinas diversas, para proporcionar una educación más equitativa, justa y que permita a los estudiantes indígenas ampliar sus oportunidades y alcanzar relaciones más igualitarias.

Hay que tener mucho cuidado de no cerrarse únicamente a los conocimientos de la comunidad, ya que se correría el riego de marginar más a los grupos indígenas, como lo reconocen los propios alumnos y los padres de familia. Por otra parte, el modelo exige a los educadores ser sensibles y flexibles a la forma de vida y de organización de las comunidades, debiendo mantener un diálogo constante con la comunidad y autoridades municipales para fomentar el interés por el bien común. Sin embargo, debe de haber una mayor apertura hacia el exterior para no generar automarginación o autoexclusión, constituyéndose en un reto para los planificadores y los coordinadores de las SC.

Otro reto importante es la integración de contenidos nacionales, ya 
que aunque valorar los conocimientos locales es importante, también se requiere formar a los estudiantes en contenidos que les permitan integrarse a otros espacios nacionales e internacionales. Sobre este aspecto, los diseñadores de la propuesta muestran sus reservas hacia el curriculum nacional que consideran como homogeneizante. Analizar este aspecto es esencial ya que no incluirlo resultaría contradictorio con la perspectiva intercultural, la cual señala la necesidad de fortalecer la cultura propia al mismo tiempo que se forma para interactuar con otras culturas. Estos aspectos son necesarios para que los alumnos puedan explicar la realidad social y cultural de las diversas comunidades a las que, en algún momento, ya sea por razones de formación o laboral, se van a integrar, especialmente si consideramos que en el siglo XXI las sociedades y comunidades son cada vez más heterogéneas (Banks 2007).

Pese a lo anterior, el modelo de Secundaria Comunitaria constituye indudablemente una alternativa para los pueblos originarios de Oaxaca; una alternativa que fomenta, en un contexto de libertad, la adquisición tanto de conocimientos formales como de conocimientos propios del ámbito comunitario; promoviendo además la reflexión, el respeto y el compromiso de los jóvenes con sus comunidades. Al plantear la necesidad de una constante reflexión sobre la educación, su quehacer, sobre sus alcances y logros, se presenta como un modelo dinámico, en constante enriquecimiento para lograr sus objetivos. 


\section{Notas}

${ }^{1}$ Los nombres de los entrevistados se omiten por respeto al anonimato y solo se utilizan siglas para referirse a ellos.

${ }^{2}$ La Coordinación General de Educación Intercultural Bilingüe (CGEIB) de la Secretaría de Educación Pública tiene, entre otras, las siguientes atribuciones: “1) Promover y evaluar la política intercultural bilingüe en coordinación con las diferentes instancias del Sistema Educativo Nacional; 2) Promover la participación de las entidades federativas y municipios, así como de los diferentes sectores de la sociedad, pueblos y comunidades indígenas, en el desarrollo de la educación intercultural bilingüe; 3) Promover y asesorar la formulación, implantación y evaluación de programas innovadores de educación intercultural bilingüe en materia de: a) Desarrollo de modelos curriculares que atiendan la diversidad, b) La formación del personal docente, técnico y directivo, c) El desarrollo y difusión de las lenguas indígenas, d) La producción regional de materiales en lenguas indígenas y e) La realización de investigaciones educativas”. Extracto del Artículo II del Acuerdo por el que se establece la Coordinación General de Educación Intercultural Bilingüe, publicado en el Diario Oficial de la Federación, 22 de enero de 2001, México. Disponible en: http://eib.sep.gob.mx/cgeib/wp-content/uploads/ 2013/03/acuerdo_creacion_cgeib.pdf /

${ }^{3}$ Las SC funcionan con una matrícula pequeña. Los grupos son de 10 a 15 alumnos por grado, atendidos por un coordinador.

${ }^{4}$ El IEEPO es la instancia gubernamental que regula la educación en el Estado de Oaxaca, México. Las Secundarias Comunitarias las autoriza el IEEPO, a partir de acuerdos políticos con el Sindicato Nacional de Trabajadores de la Educación - Sección 22 del Estado de Oaxaca, y con el apoyo de la CGEI.

${ }^{5}$ Cabe señalar que el MP ha estado presente en la educación de México en otros niveles educativos, como en el Plan y Programa de Educación Preescolar de 1993 que funcionó hasta 2004. 


\section{Bibliografía}

Ausubel, David (1976), La educación y la estructura del conocimiento, Ateneo, Buenos Aires.

Banks, James (2007), Educating citizens in a multicultural society, Teacher College, New York.

Bonfil Batalla, Guillermo (1982), "El Etnodesarrollo: sus premisas jurídicas, políticas y de organización”, en Guillermo Bonfil Batalla et al., América Latina: Etnodesarrollo y Etnocidio, Ediciones FLACSO, San José, Costa Rica, pp. 133-145.

Dewey, John (1998), Democracia y educación, Morata, Madrid.

Dietz Gunther y Cortés, Mateos (2011), Interculturalidad y educación intercultural en México: Un análisis de los discursos nacionales e internacionales en su impacto en los modelos educativos mexicanos, SEP, México.

Freire, Paulo (2007), ¿Extensión o comunicación? La concientización en el medio rural, Siglo XXI Editores, México.

Freire, Paulo (1999), La importancia de leer y el proceso de liberación, Siglo XXI Editores, México.

IEEPO (2004), Secundaria para la atención de comunidades pertenecientes a los pueblos originarios del estado de Oaxaca, Instituto Estatal de Educación Pública de Oaxaca, México.

INEGI (2010), Censo de Población y Vivienda, México.

Instituto Tecnológico y de Estudios Superiores de Monterrey (s/f), El método de proyectos como técnica didáctica, Documento elaborado para el Taller sobre el Método de Proyectos como técnica didáctica, ITESM Dirección de Investigación y Desarrollo Educativo, Vicerrectoría Académica, México.

Jiménez, Yolanda (2009), Cultura comunitaria y escuela intercultural: más allá de un contenido escolar, CGEIB, México.

Maldonado, Benjamín (2002), Autonomía y comunalidad india: enfoques y propuestas desde Oaxaca, Secretaría de Asuntos Indígenas, México.

Meyer, Lois M. (2010), “Hacia una alternativa de formación indígena bilingüe e intercultural. Una pedagogía liberadora desde la comunidad”, en Revista Latinoamericana de Educación Inclusiva, Universidad Central de Chile, Santiago de Chile, Vol. 4, N¹, pp. 25-44. 
Pérez, Enrique y Sánchez, José (2005), “La educación comunitaria: Una concepción desde la Pedagogía de la Esperanza de Paulo Freire”, Revista Venezolana de Ciencias Sociales, Universidad Nacional Experimental Rafael María Baralt, Vol. 9, N², pp. 317-329.

Quiroz, Elena (2005), “Educación intercultural para la convivencia de todos en Perú”, en Ornelas, Carlos (Coord.), Buenas Prácticas de Educación Básica en América Latina, Tomo I y II, Instituto Latinoamericano de la Comunicación Educativa, México.

Rangel, Alfonso (2006), “La educación rural mexicana y la educación fundamental en el inicio del CREFAL”, en Revista Interamericana de Educación de Adultos, CREFAL, México, Vol. 28, N², pp. 169-176.

Ruiz, Arturo (2002), “Una breve revisión de multiculturalidad y la educación multicultural o intercultural”, en Héctor Muñoz (Coord.), Rumbo a la interculturalidad, UPN-UABJO-UAM-I, México.

-Idem (1993), Educación Indígena: del discurso a la práctica docente, UABJO, México.

Sánchez, Javier (2008), Manual del Educador Comunitario: Proyectos de Aprendizaje, Documento de Trabajo, Oaxaca, México.

Recibido: 15.05.2014

Aceptado: 15.07.2014 